\title{
Magnetoimpedance Effects in Electrodeposited NiFe Nanowire Array
}

\author{
E. Aydogmus, H. Kaya, F.E. Atalay, S. Atalay and D. Avcu \\ Inonu University, Science and Art Faculty, Department of Physics, Malatya 44280, Turkey
}

\begin{abstract}
In this work, $\mathrm{NiFe}$ nanowires were grown in highly ordered porous anodic alumina oxide templates by dc electrodeposition at a $\mathrm{pH}$ value of 2.6. Scanning electron microscopy showed that wires have diameters of about 250 $310 \mathrm{~nm}$ and length $25-30 \mu \mathrm{m}$. The energy dispersive X-ray analysis showed that the composition of the nanowires is $\mathrm{Ni}_{65} \mathrm{Fe}_{35}$. Electrical contacts were made on both sides of the nanowire array and their magnetoimpedance properties were investigated. All the magnetoimpedance curves showed single peak behavior due to the high shape anisotropy. The maximum magnetoimpedance change at the $79 \mathrm{MHz}$ driving current frequency was $\approx 1.45 \%$.
\end{abstract}

DOI: $10.12693 /$ APhysPolA.125.227

PACS: 81.07.Gf, 81.15.Pq, 75.75.-c

\section{Introduction}

The magnetoimpedance (MI) effect can be defined as the variation of the impedance of a magnetic material under the effect of a dc magnetic field, usually applied along the sample length. There has been a considerable upsurge of interest in the MI effect found in soft, amorphous, ferromagnetic materials and films due to wide range of application of these sensors in technology [1-3].

Magnetoimpedance properties of amorphous wires, ribbons, composite $\mathrm{NiFe}-\mathrm{CoNiFe}$ wires and magnetic films have been studied widely [1-5]. Ciureanu et al. [6] studied GMI effect in soft NiFe-permalloy and ultrasoft NiCo-based amorphous fibers and in permalloy wires. Both longitudinal and transverse GMI effects had been observed in these fibers. Beach and Berkowitz [1] studied MI effect in NiFe plated wire. Hika et al. [7] had presented results on $\mathrm{MI}$ in a $\mathrm{NiFe} / \mathrm{Co} / \mathrm{NiFe}$ sandwich film of $0.2 \mu \mathrm{m}$ thickness, which exhibited the impedance changes up to $8 \%$ for a field of 4 Oe at a frequency of $400 \mathrm{MHz}$. Mohri et al. [2] presented new high performance micro magnetic heads and sensors utilizing the $\mathrm{MI}$ in the Co-rich amorphous wire.

MI has made it possible to construct new micro-sized magnetic field sensors with a high sensitivity and quick response, which are expected in the near future to replace giant magnetoresistive elements and fluxgate sensors. Basically magnetoimpedance studies have two aims; one is to increase sensitivity and the other one is to reduce the size of magnetoimpedance sensor. In this study we aim to reduce size of MI sensor and for this reason, MI properties of NiFe nanowire array were investigated.

\section{Experimental}

A three-electrode cell was used for the nanowire electrodeposition. The volume of the electrochemical bath was approximately $50 \mathrm{ml}$. $\mathrm{An} \mathrm{Ag} / \mathrm{AgCl}$ electrode (BAS, $3 \mathrm{M} \mathrm{NaCl}$, and $-35 \mathrm{mV}$ versus $\mathrm{SCE}$ at $25^{\circ} \mathrm{C}$ ) was used as the reference electrode. A platinum electrode approximately 5 times larger than the cathode was used as an auxiliary electrode. Anodic alumina oxide (AAO) membranes, with specified pore diameter of $200 \mathrm{~nm}$ and pore length of $60 \mu \mathrm{m}$, were used as the cathode for the nanowire synthesis. These have an exposed area of approximately $1 \mathrm{~cm}^{2}$. The membranes were supplied by the Whatman Company. Before the electrodeposition, one side of the AAO templates was coated with $\mathrm{Au}$ with a thickness of $10 \mathrm{~nm}$, and an adhesive carbon disc and copper foil were also stuck on for electrical contact [8]. The bath contained a mixed solution of $0.2 \mathrm{M}$ $\mathrm{Ni}\left(\mathrm{SO}_{4}\right)_{2} \cdot 6 \mathrm{H}_{2} \mathrm{O}, 5 \mathrm{mM} \mathrm{Fe}\left(\mathrm{SO}_{4}\right)_{2} \cdot 6 \mathrm{H}_{2} \mathrm{O}, 0.2 \mathrm{M} \mathrm{H}_{3} \mathrm{BO}_{3}$, $35 \mathrm{mM} \mathrm{NaCl}$ and $0.1 \mathrm{~g} / 1$ sodium lauryl sulfate. All solutions were prepared by dissolving reagent-grade chemicals in deionised water. The bath $\mathrm{pH}$ was adjusted to 2.6 by adding $0.1 \mathrm{M} \mathrm{HCl}$ or $0.1 \mathrm{M} \mathrm{NaOH}$, using a Jenway $3520 \mathrm{pH}$ meter. The electrodeposition was carried out at $25^{\circ} \mathrm{C}$ in a stirred solution and at a constant potential of $-2 \mathrm{~V}$ versus $\mathrm{Ag} / \mathrm{AgCl}$ for 180 min to produce the nanowires. The electrodeposition of $\mathrm{NiFe}$ was performed by means of an electrochemical analyzer system, an Iviumstat potentiostat/galvanostat.

The morphology of the nanoarrays was investigated by scanning electron microscopy (JEOL JSEM 7001F). The quantitative chemical analyses of the alloys were performed by energy dispersive X-ray (EDX) spectroscopy.

Samples were connected to the impedance measurement circuit using silver conductive paint. The MI was measured in an axial magnetic field using a Hewlett Packard 4294A impedance analyser with a 42941A impedance probe. MI data were obtained for a range of frequencies up to $80 \mathrm{MHz}$ using a constant-amplitude $\mathrm{AC}$ driving current of $1 \mathrm{~mA}$. The MI ratio was calculated from the equation $\Delta Z / Z[\%]=\left[\left(Z(H)-Z_{\max }\right) / Z_{\max }\right] \times$ 100 , where $Z_{\max }$ is the impedance measured at a magnetic field of $H \approx 1 \mathrm{~T}$. Magnetic field was applied along the wire length direction using an electromagnet in MI measurements.

\section{Results and discussions}

The NiFe nanowires were produced in AAO templates by electrodeposition method. After the dissolution of 
the template in $1 \mathrm{M} \mathrm{NaOH}$ for three days, nanowires were separated from AAO template and SEM picture were obtained (Fig. 1). It can be seen that the diameter and length of nanowires are about 250-310 nm and $29 \mu \mathrm{m}$, respectively. The EDX analysis showed that the composition of the nanowires is $\mathrm{Ni}_{65} \mathrm{Fe}_{35}$. It was found that after 180 min deposition time, the AAO was fully filled with $\mathrm{NiFe}$ and small $\mathrm{NiFe}$ islands start to form on the surface of AAO template. The top surface of AAO template was coated with $\mathrm{Au}$ film and in this way whole side of the AAO template was coated with Au film, since the other side of AAO template was previously coated with $\mathrm{Au}$ film for the production process of nanowire.
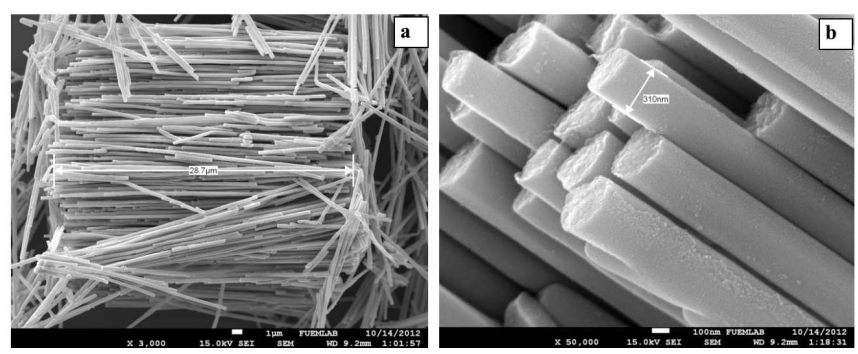

Fig. 1. (a) SEM images of ordered NiFe nanowires, (b) higher magnification SEM image.

Finally, electrical contacts, which were connected to impedance analyser, were made to the Au films using silver conductive paint. The variation of impedance value of $\mathrm{NiFe}$ nanowire array is given in Fig. 2, as it can be seen the impedance value increases with increasing driving current frequency showing that the skin effect is dominant for nanowire array. The skin effect has a classical electromagnetic origin, being related to the magnetic penetration depth $\delta$, which is given in Eq. (1) [1-3]:

$$
\delta=\left(\frac{2}{\omega \sigma \mu}\right)^{1 / 2},
$$

where $\sigma$ is the conductivity, $\omega$ is the AC current frequency and $\mu$ is the permeability. It has been shown that the impedance $Z$ is related to the skin depth through the following expression [1-3]:

$$
Z=R_{\mathrm{dc}} j k t \operatorname{coth}(j k t), \quad k=(1+j) / \delta,
$$

where $R_{\mathrm{dc}}$ is the dc resistance and $t$ - the thickness of the magnetic sample. For soft magnetic materials, the permeability can achieve very high values, which, associated with relatively large frequencies, can lead to penetration depths smaller than the wire radius. In these cases, the applied current flows effectively just in a shell near the surfaces [3], giving rise to increased values of the total ac impedance. The net effect of an applied longitudinal dc field is to reduce the value of the transverse permeability, increasing the penetration depth, and consequently reducing the value of the measured impedance. MI results from a decrease in the impedance response of a ferromagnetic material subjected to an ac current, when a dc magnetic field is applied.

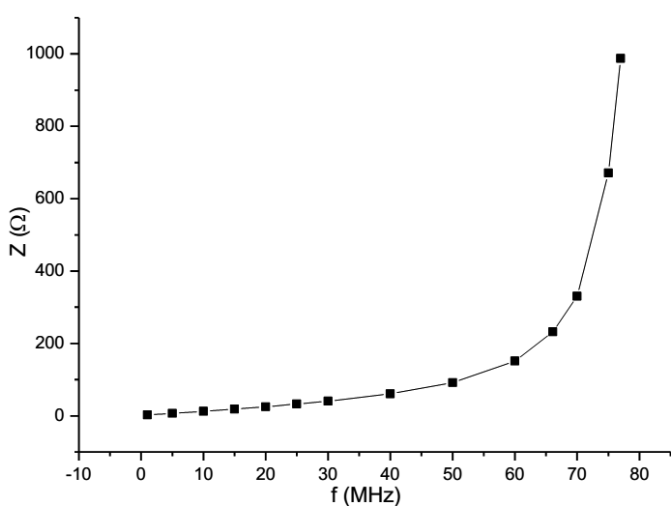

Fig. 2. Impedance variation as a function of driving current frequency at $H=0 \mathrm{~A} / \mathrm{m}$.

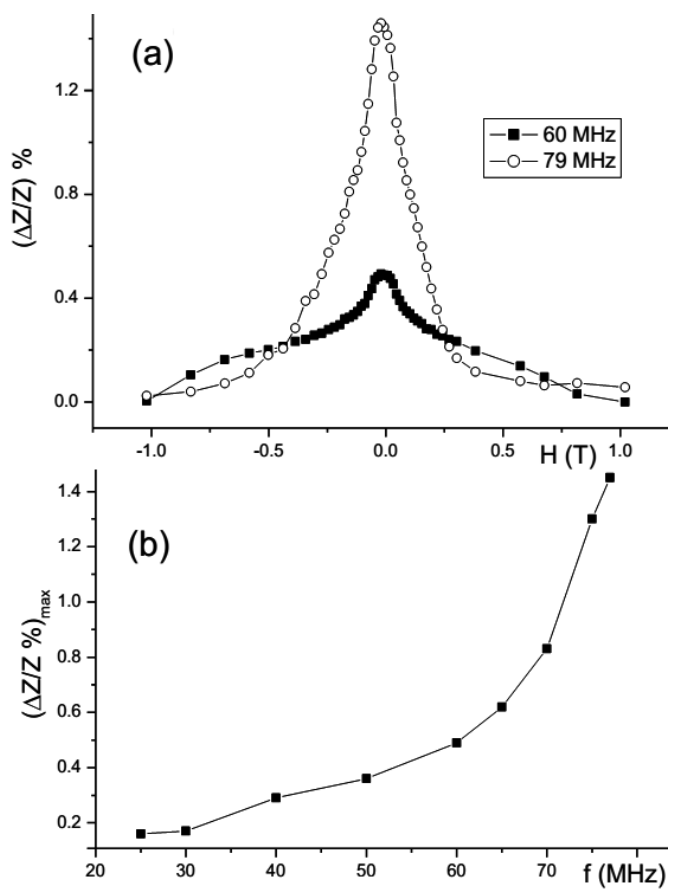

Fig. 3. (a) MI curves of NiFe nanowire arrays at $60 \mathrm{MHz}$ and $79 \mathrm{MHz}$, (b) maximum MI changes as a function of driving current frequency.

Effect of driving current frequency on the MI response is shown in Fig. 3. MI change increases with increasing driving current frequency and reaches to a maximum value of $1.45 \%$ at $79 \mathrm{MHz}$. It has been shown that for a typical nanowire, the shape anisotropy could be of the order of $4 \times 10^{6} \mathrm{erg} / \mathrm{cm}^{3}$ [9], which is comparable with other types of anisotropy. Therefore, the maximum change in the MI effect in a nanowire is smaller than that for amorphous alloys or NiFe composite wires. Mandal and Ghatak [10] claimed that a relative change in ac resistance up to $12 \%$ in an applied field was found in Co-rich amorphous wire. This large change was ascribed to the giant magnetoresistance effect. Since the relative 
change in the real part (resistive part) is also high in $\mathrm{NiFe}$ nanowire array, it was assumed that total change in MI effect may be resulting from resistance change.

MI magnitude and curve shape were largely determined by the magnetic domain configuration and relative contributions of both domain wall motions and magnetic moment rotations to the overall magnetization process. Since all MI curves showed single peak behaviour, this shows that nanowire arrays exhibit uniaxial magnetic anisotropy with the easy magnetization direction close to the nanowire axes.

\section{Conclusions}

The NiFe nanowires were produced in AAO templates by electrodeposition method. MI change increases with increasing driving current frequency and reaches a maximum value of $1.45 \%$ at $79 \mathrm{MHz}$. Small change in MI effect was attributed to large shape anisotropy in nanowires.

\section{Acknowledgments}

This work was supported by TÜBİTAK under project number TBAG-111T761 and Inonu University with the project number I.U.A.F-2012/185.

\section{References}

[1] R.S. Beach, A.E. Berkowitz, Appl. Phys. Lett. 64, 3652 (1994).

[2] K. Mohri, L.V. Panina, T. Uchiyama, K. Bushida, M. Noda, IEEE Trans. Magn. 31, 1266 (1995).

[3] M.H. Phan, H.X. Peng, Prog. Mater. Sci. 53, 323 (2008).

[4] X.P. Li, J. Fan, J. Ding, X.B. Qian, J. Magn. Magn. Mater. 300, e98 (2006).

[5] F.E. Atalay, S. Atalay, J. Alloys Comp. 392, 322 (2005).

[6] P. Ciureanu, P. Rudkowski, G. Rudkowska, D. Menard, M. Britel, J.F. Currie, J.O. Strom-Olsen, A. Yelon, J. Appl. Phys. 79, 5136 (1996).

[7] K. Hika, L.V. Panina, K. Mohri, IEEE Trans. Magn. 32-5, 4594 (1996).

[8] F.E. Atalay, H. Kaya, V. Yagmur, S. Tari, S. Atalay, D. Avsar, Appl. Surf. Sci. 256, 2414 (2010).

[9] J.H. Gao, Q.F. Zhan, W. He, D.L. Sun, Z.H. Cheng, J. Magn. Magn. Mater. 305, 365 (2006).

[10] K. Mandal, S.K. Ghatak, Phys. Rev. B 47, 14233 (1993). 